\title{
Initial experience with the unrestricted introduction of sugammadex at a large academic medical center: a retrospective observational study examining postoperative mechanical ventilation and efficiency outcomes
}

\author{
Vikas N. O'Reilly-Shah, Grant C. Lynde, Matthew L. Mitchell, \\ Carla L. Maffeo, Craig S. Jabaley, and Francis A. Wolf \\ Department of Anesthesiology, Emory University School of Medicine, Atlanta, GA, USA
}

\begin{abstract}
Background: Sugammadex rapidly reverses deep neuromuscular blockade, but owing to cost, questions remain about its optimal utilization. After the unrestricted introduction of sugammadex at Emory University Hospital, we hypothesized that reductions would be demonstrated in the primary outcome of post-anesthesia care unit (PACU) mechanical ventilation (MV) and secondary outcomes of PACU length of stay (LOS) and emergence time (surgery end to anesthesia end time in the PACU).

Methods: This retrospective observational study included patients undergoing general anesthesia over a 12-month period. Using multiple variable penalized logistic regression in a one-group before-and-after design, we compared the categorized rates of PACU MV to examine the effect of sugammadex introduction following a post-hoc chart review to ascertain the reason for postoperative MV. Additionally, multiple variable linear regression was used to assess for differences in PACU LOS and emergence time within a propensity-matched set of patients receiving neostigmine or sugammadex. Results: In total, 7,217 surgical cases met the inclusion criteria: 3,798 before and 3,419 after sugammadex introduction. The incidence of PACU MV was $2.3 \%$ before and $1.8 \%$ after $(\mathrm{P}=0.118)$ sugammadex introduction. PACU MV due to residual neuromuscular blockade $(\mathrm{rNMB})$ decreased from $0.63 \%$ to $0.20 \%(\mathrm{P}=0.005)$. Ventilation because of other causes was unchanged. PACU LOS and emergence time were unchanged in the propensity-matched set of 1,444 patients. Conclusions: rNMB was an important contributor to PACU MV utilization and its incidence significantly decreased after sugammadex introduction. The selected efficiency measures may not have been sufficiently granular to identify improvements following introduction.
\end{abstract}

Keywords: Neostigmine; Neuromuscular blockade; Rocuronium; Sugammadex.

Corresponding author: Grant C. Lynde, M.D., M.B.A.

Department of Anesthesiology, Emory University School of Medicine, 1364 Clifton Road NE, Atlanta, GA 30322, USA

Tel: +1-253-307-9162, Fax: +1-404-778-3900, Email: glynde@emory.edu

ORCID: https://orcid.org/0000-0002-2055-3484

It was presented at ANESTHESIOLOGY 2017 Annual Meeting of The American Society of Anesthesiologists (ASA), October 2017, Boston Convention and Exhibition Center, Boston, USA.

Received: March 7, 2018. Revised: April 23, 2018 (1st); May 12, 2018 (2nd). Accepted: May 15, 2018.

Korean J Anesthesiol 2018 October 71(5): 374-385

https://doi.org/10.4097/kja.d.18.00063

(c) This is an open-access article distributed under the terms of the Creative Commons Attribution Non-Commercial License (http://creativecommons.org/ licenses/by-nc/4.0/), which permits unrestricted non-commercial use, distribution, and reproduction in any medium, provided the original work is properly cited. 


\section{Introduction}

Sugammadex is capable of directly reversing the effects of aminosteroidal neuromuscular blocking agents (NMBAs, e.g., rocuronium and vecuronium), by forming tight water-soluble complexes at a $1: 1$ ratio [1]. Compared to cholinesterase inhibitors, NMBA antagonism with sugammadex results in faster recovery and the ability to completely recover from even profound blockade, thus conferring the potential to eliminate residual neuromuscular blockade ( $\mathrm{rNMB}$ ) in routine practice [1-7]. Between $16-60 \%$ of patients reversed with neostigmine will demonstrate signs of $\mathrm{rNMB}$, contributing to morbidity from postoperative pulmonary complications (PPCs), such as hypoxemia, airway obstruction, and pneumonia [5,6,8]. Owing to the limited efficacy of cholinesterase inhibitors, some patients cannot be safely extubated at the conclusion of surgery, either because of rNMB following attempted antagonism or blockade that is too profound to even attempt antagonism $[9,10]$. This could beget increased utilization of operating room time after the conclusion of surgery or the decision to unexpectedly employ mechanical ventilation (MV) in the post-anesthesia care unit (PACU).

Although sugammadex has been available globally since 2008, it did not receive United States (US) Food and Drug Administration approval until December 2015. As a result, US practitioners, practice groups, and hospitals must decide if and how to incorporate sugammadex during routine clinical practice, which is influenced in part by its high direct costs [11]. However, there may be opportunities for indirect savings. For example, postoperative $\mathrm{MV}$ is associated with longer PACU stays and increased financial burden $[5,12,13]$. A decrease in postoperative MV would be expected to result in lessened PACU length of stay (LOS) and decreased provider workload, favorably impacting the pharmacoeconomic analyses underpinning sugammadex utilization decisions $[10,14]$. Similarly, the ability of sugammadex to completely reverse NMB at nearly any level could result in more rapid intraoperative recovery times.

We sought to examine practical outcomes after the introduction of sugammadex for unrestricted use by anesthesia providers at Emory University Hospital. We hypothesized that there would be a reduction in the primary outcome of PACU MV. Likewise, we anticipated reductions in the secondary outcomes of PACU LOS and the time from surgery stop to anesthesia stop ("emergence time").

\section{Materials and Methods}

We conducted a retrospective observational study of routinely recorded data between October 1, 2015 and October 1, 2016 to examine the clinical and efficiency outcomes following the unrestricted introduction of sugammadex into practice at Emory University Hospital. This study was approved by the Emory University Institutional Review Board (\#IRB00092068), including a waiver of informed consent given minimal risk. This manuscript conforms with the applicable EQUATOR checklist for observational studies (STROBE).

\section{Outcomes}

One primary outcome was specified a priori: (1) the rate of PACU MV. Three secondary outcomes were analyzed. The first two were specified a priori: (2) PACU LOS and (3) emergence time. As discussed subsequently, an additional secondary outcome was specified post-hoc: (4) rate of PACU MV by cause. PACU LOS was defined as the time between anesthesia end time and readiness for PACU discharge as is routinely documented.

\section{Design}

The design was quasi-experimental with a one-group pre/ post design for outcomes (1) and (4) and multiple variable regression with propensity score matching for outcomes (2) and (3). The study period was chosen to allow for the assessment of a sufficient number of ventilated patients while maintaining the feasibility of manual chart review to identify a cohort for outcomes (1) and (4); a formal power analysis was not performed.

The study was divided into two time periods: six and a half months before (P1, October 1, 2015 to April 12, 2016) and five and a half months after (P2, April 13, 2016 through October 1, 2016) sugammadex was made available. Once added to the hospital formulary, sugammadex was supplied as part of a standard medication tray for every surgical case without restrictions on provider use. Prior to introduction, providers were educated about its use. Neostigmine and glycopyrrolate were included in the same tray. Choice of NMBA antagonist was at the discretion of the care team. Qualitative nerve monitors were available for all surgical cases, and quantitative monitors (i.e., accelerometers) were available on request.

\section{Data acquisition}

Using structured queries against the Emory Healthcare Clinical Data Warehouse (CDW), we identified for inclusion all surgical cases performed using general endotracheal anesthesia (GETA), had an electronic anesthesia record during the study period, and had recovered in the PACU. Patients who were already intubated prior to their procedure and those who did not recover in the PACU (direct ICU admission) were excluded. For each patient, age, sex, height, weight, American Society of Anesthesiologists physical status classification (ASA-PS), glomerular 
filtration rate, and surgical specialty data were extracted from the CDW. NMBAs and antagonists used, as well as timestamps for anesthesia and surgery start/stop, PACU admission time, and routinely recorded readiness for PACU discharge were also extracted. For outcome (2), PACU LOS reflected the span of time between admission to the PACU and readiness for discharge, not the time when patients physically left the PACU. With regards to outcome (3), some patients were ready for transport from the operating room to the PACU, but PACU staff were not available to assume care of the patient. The cases of such patients are flagged with a "PACU hold" time per protocol at our institution. The time between surgery end and the PACU hold time was used for outcome (3) in these instances.

\section{Data validation and review}

First-pass identification of patients with possible mechanical ventilation in the PACU was performed by identifying within the CDW respiratory therapy interventions (i.e., documented ventilation mode) during the PACU phase of care. This approach was validated against known cases of PACU mechanical ventilation. Further confirmation of PACU mechanical ventilation was made using a detailed manual chart review by two investigators (CM and $\mathrm{MM}$ ). After obtaining the results from the analysis of outcome (1), a post-hoc determination of the reason for PACU MV was undertaken on a per-case basis by two investigators (CM and MM). These causes were assigned to categories, subject to review and confirmation by two additional investigators (GL and VO), who also refereed disagreements. This analysis led to the examination of secondary outcome (4). In addition to a major category assignment, one or more subcategories were assigned to each. Specifically, for categorization of PACU MV due to rNMB, which was defined as any of the following: documentation of train-of-four (TOF) $<4$ at case end, documentation of TOF with subjective fade at case end, documentation of TOF ratio $<0.9$ (by accelerometry) at case end, or free-text documentation of rNMB by the intraoperative or PACU anesthesia providers.

\section{Statistical analysis}

Following the identification of surgical cases with complete data from P1 and P2, further statistical analysis was performed using R v 3.3 (R Core Team, Austria) [15]. A significance level a of 0.05 was used for all statistical tests. For single variable analysis, categorical data were analyzed using chi-squared tests, while continuous data were analyzed using $t$-tests (except for case length, which was analyzed using the Wilcoxon rank-sum test), using functions included in the base installation of $\mathrm{R}$.

The dependent variables included (1) a binomial categori- zation PACU MV, (2) the logarithm of PACU LOS time, (3) the logarithm of emergence time, and (4) binomial categorization of PACU MV by cause. The independent variables included patient age, sex, height, log weight, categorized ASA-PS (1/2, 3, 4/5, or Emergency [any]), categorized estimated glomerular filtration rate $\left(<30,30-60\right.$, or $\left.>60 \mathrm{ml} / \mathrm{min} / \mathrm{m}^{2}\right)$, surgical specialty type, and log case length (time from surgery start to surgery stop as documented in the intraoperative record). Data were manually inspected, transformed to normality if necessary, and outlier cases were removed. Baseline distribution and transformation to normality, if performed, were assessed graphically by inspecting quantile-quantile plots generated using a normal distribution to model theoretical quantiles. The exclusion criteria and number of cases excluded are shown in Fig. 1. Height displayed satisfactory characteristics of normal distribution without transformation. Age was not normal in distribution, and uncomplicated transformations via root or logarithm did not yield improvement. Age was therefore left untransformed for regression analysis [16]. Cases with missing data for any of the dependent variables were excluded from the complete case regression analysis. A dummy dependent variable (Era) was included in both types of regression modeling described below, where Era $=0$ was assigned for P1 and Era $=1$ was assigned for P2.

Owing to the relatively low incidence of the binomial outcomes of interest (1) and (4), resulting in complete separation in some of the categorical control variables, a penalized-likelihood logistic regression analysis was performed using Firth's method via the "logistf" package in R [17-19]. Sensitivity analysis was performed using standard logistic regression by collapsing certain categorical control variables that had complete separation without this combination of categories. Interrupted time series analysis including the time factor was not attempted because of the rarity of the events in the time series. Likelihood ratio tests were performed to determine the significance of the contribution of each dependent variable included in the model.

For outcomes (2) and (3), we analyzed only cases in P2. We assigned an additional dummy variable to each of these cases to indicate whether they had received only neostigmine or only sugammadex. Cases not administered either of these drugs, or both, were excluded. We then performed propensity matching using the genetic algorithm within the "MatchIt" $\mathrm{R}$ package [20,21]. As described by Ho et al. [21], this approach uses "a genetic search algorithm to find a set of weights for each covariate such that optimal balance is achieved after matching." This methodology samples with replacement to optimize the global propensity score, and searches for an optimal matched pool from both the "treatment" group (sugammadex) and the "control" group (neostigmine) rather than finding a $1: \mathrm{m}$ ratio matched group. For sensitivity analysis, other algorithms for matching were also employed (greedy nearest neighbor, optimal, 


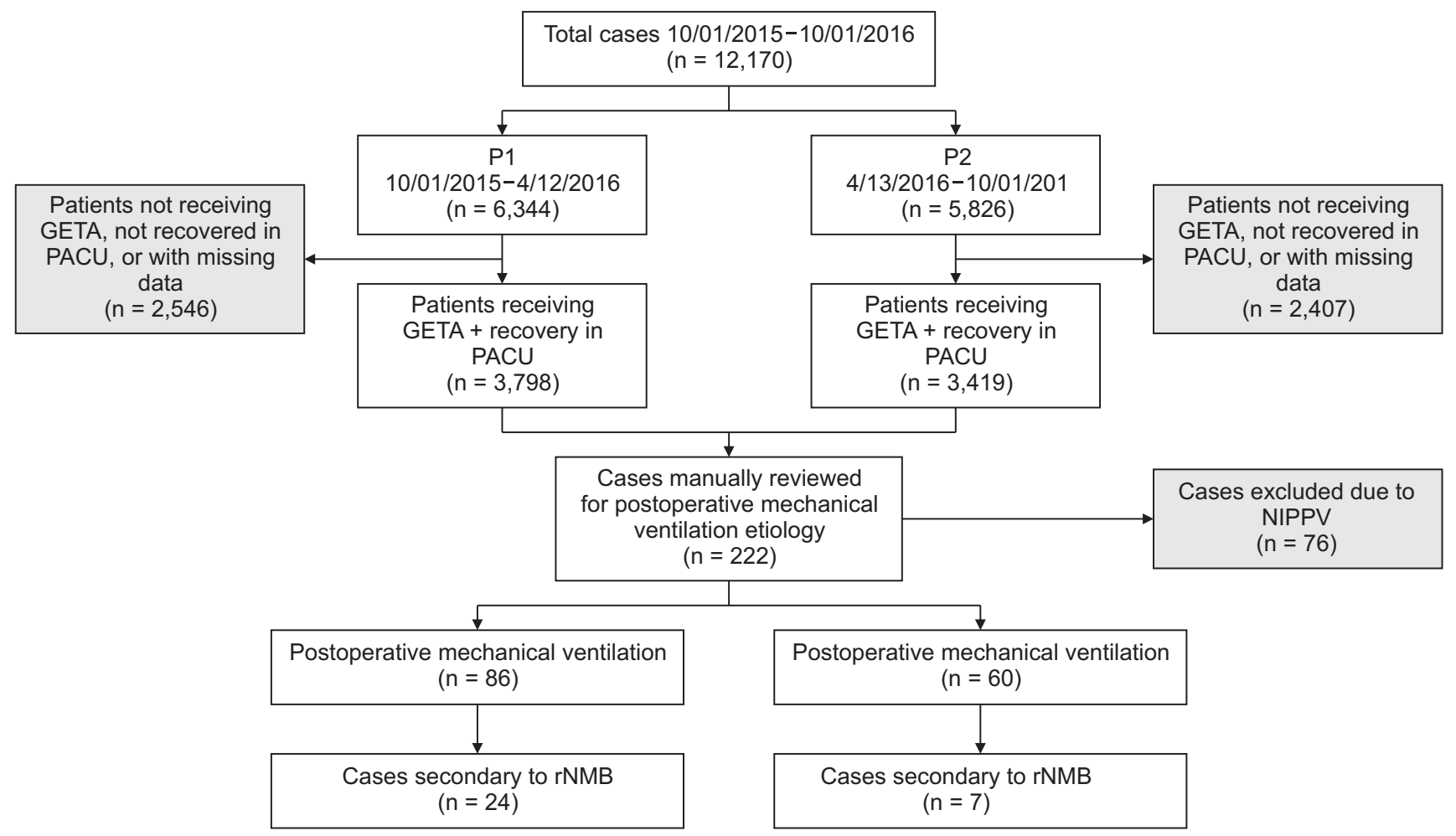

Fig. 1. Study profile with case numbers, exclusions, and main findings related to mechanical ventilation in the post-anesthesia care unit. All patients to the left of center are from P1, and all patients to the right of center are from P2. GETA: general endotracheal anesthesia, PACU: post-anesthesia care unit, NIPPV: non-invasive positive pressure ventilation, rNMB: residual neuromuscular blockade.

and coarsened exact matching). Matching was performed on the following independent variables: age, sex, weight, ASA-PS, renal function categorization, case length, and surgical specialty. Owing to residual imbalance, we performed multiple variable ordinary least squares linear regression by using the entire cohort as well as the matched samples. This was performed using the $g l m$ function of the "stats" package in R, included in the base installation. The dependent variables in these analyses were outcomes (2) and (3). The independent variables were those described for matching as well as the dummy variable for neostigmine/sugammadex. Emergence times between $2 \mathrm{~min}$ and 150 min were included in that regression analysis. Because we were working with log-transformed outcomes of interest, following linear regression, the resultant coefficients were exponentiated and adjusted as $\widehat{\beta l, a d} \jmath=100 *(\exp (\widehat{\beta} \iota)-1)$ to determine the estimate of the percentage change associated with (a) each categorical independent variable compared to the referent or (b) with a one-unit change in continuous independent variables [22]. This was a reasonable approximation for a $1 \%$ change in our log-transformed dependent variables as well.

\section{Results}

\section{Patient characteristics}

There were 4,778 cases in P1 and 4,337 in P2 who received GETA. After excluding cases not recovered in the PACU and those with missing data, 3,798 cases in P1 and 3,419 in P2 were included in the final analytic dataset. Characteristics of the cases analyzed are summarized in Table 1 . The majority of patients were female, had ASA-PS 3, and represented a range of surgical specialties, the most common being general and oncologic surgery. The patient populations and case characteristics were similar in both time periods for all of the examined characteristics except median height $(167.6 \mathrm{~cm}$ vs. $170.2 \mathrm{~cm}, \mathrm{P}=0.035)$ and case length (109 min vs. $105 \mathrm{~min}, \mathrm{P}=0.008$ ).

\section{NMBA and antagonist agents}

Nearly all patients undergoing GETA received a NMBA in $\mathrm{P} 1$ and $\mathrm{P} 2$, with rocuronium being used in a majority of cases in both $\mathrm{P} 1$ and $\mathrm{P} 2$. The use of vecuronium and succinylcholine was lower in P2 by a small but statistically significant margin (Table 1). Some cases received more than one NMBA, includ- 
Table 1. Patient, Surgical Case, and Neuromuscular Blocking/Reversal Agent Use Characteristics before (P1) and after (P2) Sugammadex Availability amongst those Receiving General Endotracheal Anesthesia with Recovery in the PACU

\begin{tabular}{|c|c|c|c|}
\hline & $\mathrm{P} 1(\mathrm{n}=3,798)$ & $\mathrm{P} 2(\mathrm{n}=3,419)$ & $P$ value \\
\hline Age $(y r)^{*}$ & $55.1 \pm 16.1$ & $54.4 \pm 15.9$ & 0.062 \\
\hline Sex $(\text { Female })^{\ddagger}$ & $2,151(56.6 \%)$ & $1,925(56.3 \%)$ & 0.795 \\
\hline Height $(\mathrm{cm})^{*}$ & $167.6(131.2-208.3)$ & $170.2(134.5-203.2)$ & 0.035 \\
\hline Weight $(\mathrm{kg})^{*}$ & $79.4(31.3-198.6)$ & $79.5(30-231.8)$ & 0.245 \\
\hline $\operatorname{GFR}\left(\mathrm{ml} / \mathrm{min} / \mathrm{m}^{2}\right)^{\ddagger}$ & & & 0.442 \\
\hline$<30$ & $313(8.2 \%)$ & $285(8.3 \%)$ & \\
\hline $30-60$ & $451(11.9 \%)$ & $439(12.8 \%)$ & \\
\hline$>60$ & $3,034(79.9 \%)$ & $2,695(78.8 \%)$ & \\
\hline ASA classification $^{\ddagger}$ & & & 0.059 \\
\hline $1 / 2$ & $1,227(32.3 \%)$ & $1,070(31.3 \%)$ & \\
\hline 3 & $2,089(55 \%)$ & $1,882(55 \%)$ & \\
\hline $4 / 5$ & $307(8.1 \%)$ & $316(9.2 \%)$ & \\
\hline Emergency (any) & $175(4.6 \%)$ & $151(4.4 \%)$ & \\
\hline Case length $(\min )^{\dagger}$ & $109(56-177)$ & $105(50-176)$ & 0.034 \\
\hline Surgical specialty $^{\ddagger}$ & & & 0.094 \\
\hline Cardiac/thoracic/vascular & $481(12.7 \%)$ & $439(12.8 \%)$ & \\
\hline General/oncology & $1,281(33.7 \%)$ & $1,111(32.5 \%)$ & \\
\hline Neurosurgery & $614(16.2 \%)$ & $494(14.4 \%)$ & \\
\hline Urology & $518(13.6 \%)$ & $488(14.3 \%)$ & \\
\hline Gynecology & $224(5.9 \%)$ & $208(6.1 \%)$ & \\
\hline Gastroenterology & $410(10.8 \%)$ & $436(12.8 \%)$ & \\
\hline Other & $270(7.1 \%)$ & $243(7.1 \%)$ & \\
\hline Neuromuscular blocking agents ${ }^{\ddagger}$ & $3,713(97.8 \%)$ & $3,330(97.4 \%)$ & 0.351 \\
\hline Rocuronium & $2,917(76.8 \%)$ & $2,623(76.7 \%)$ & 0.954 \\
\hline Vecuronium & $431(11.3 \%)$ & $334(9.8 \%)$ & 0.033 \\
\hline Cisatracurium & $265(7.0 \%)$ & $219(6.4 \%)$ & 0.356 \\
\hline Succinylcholine & $1,091(28.7 \%)$ & $892(26.1 \%)$ & 0.013 \\
\hline None & $85(2.2 \%)$ & $89(2.6 \%)$ & 0.351 \\
\hline Received any $\mathrm{NMBA}^{\ddagger}$ & $3,185(83.9 \%)$ & $2,874(84.1 \%)$ & 0.842 \\
\hline NMBA and neostigmine & $2,886(90.6 \%)$ & $883(30.7 \%)$ & $<0.001$ \\
\hline NMBA and sugammadex & $0(0 \%)$ & $1,767(61.5 \%)$ & $<0.001$ \\
\hline NMBA and no reversal & $299(9.4 \%)$ & $238(8.3 \%)$ & 0.142 \\
\hline
\end{tabular}

Values are expressed as mean \pm SD or number (\%) or median (IQR). GFR: glomerular filtration rate, ASA: American Society of Anesthesiologists, NMBA: nondepolarizing neuromuscular blocking agent. P values calculated using t-tests ( ${ }^{*}$ continuous), Wilcoxon rank-sum $\left({ }^{\dagger}\right.$ case length), or chisquared analysis ( ${ }^{\ddagger}$ categorical). Totals may exceed $100 \%$ as some patients received multiple agents.

ing multiple nondepolarizing agents. While the percentage of cases given any antagonist (neostigmine, sugammadex, or both) following a nondepolarizing NMBA was $>90 \%$ in both P1 and $\mathrm{P} 2$, neostigmine use dropped from $90.6 \%$ to $30.7 \%(\mathrm{P}<0.001)$, representing a two-thirds decrease following the introduction of sugammadex. Sugammadex became the antagonist of choice after its introduction and was used in $61.5 \%$ of cases receiving NMBA during P2. A small number of cases $(n=14)$ received both neostigmine and sugammadex during P2, suggesting sugammadex was used as a rescue agent following attempted NMBA antagonism with neostigmine.

\section{MV in the PACU}

The rates and causes of PACU MV are shown in Table 2. Major causes included respiratory/airway, hemodynamic, neurological, or iatrogenic ones, as well as rNMB and lack of intensive care bed (did not plan to extubate) (See Supplemental Appendix, Table S1, for additional detail). The overall incidence of PACU MV was 86 of 3,798 cases (2.3\%) in P1 and 60 of 3,419 (1.8\%) in P2. Multiple variable penalized-likelihood logistic regression using Firth's method demonstrated no overall difference after accounting for the control variables. We proceeded to perform an analogous multiple variable regression analysis against each of the identified overall reasons for PACU MV. The results of these regression analyses, reporting only the significance of the difference between $\mathrm{P} 1$ and P2, are also shown in Table 2. The 
Table 2. Rates and Causes of Post-anesthesia Care Unit Mechanical Ventilation before (P1) and after (P2) the Introduction of Sugammadex into Clinical Care

\begin{tabular}{lccccc}
\hline \multicolumn{1}{c}{$\begin{array}{c}\text { Cause of PACU } \\
\text { mechanical ventilation }\end{array}$} & Total & Pre (P1) & Post (P2) & $\begin{array}{c}\text { Odds ratio of PACU } \\
\text { mechanical ventilation in the } \\
\text { post-introduction era versus the } \\
\text { pre-introduction era (95\% CI) }\end{array}$ \\
\hline Did not plan to extubate & $32(21.9 \%)$ & $19(22.1 \%)$ & $13(21.7 \%)$ & $0.772(0.373-1.551)$ & 0.469 \\
Hemodynamic & $18(12.3 \%)$ & $6(7.0 \%)$ & $12(20.0 \%)$ & $2.030(0.807-5.616)$ & 0.134 \\
Iatrogenic & $16(11.0 \%)$ & $11(12.8 \%)$ & $5(8.3 \%)$ & $0.546(0.182-1.451)$ & 0.230 \\
Neurological & $18(12.3 \%)$ & $11(12.8 \%)$ & $7(11.7 \%)$ & $1.124(0.557-2.282)$ & 0.622 \\
Respiratory & $31(21.2 \%)$ & $15(17.4 \%)$ & $16(26.7 \%)$ & 0.108 & $0.339(0.139-0.736)$ \\
rNMB & $31(21.2 \%)$ & $24(27.9 \%)$ & $7(11.7 \%)$ & $0.938(0.645-1.358)$ & 0.733 \\
All except rNMB & $115(78.8 \%)$ & $62(72.1 \%)$ & $53(88.3 \%)$ & $0.767(0.547-1.069)$ & 0.118 \\
All causes & 146 & 86 & 60 & & \\
\hline
\end{tabular}

Values are expressed as number (\%). PACU: post-anesthesia care unit, rNMB: residual neuromuscular blockade. P values were derived by performing multiple variable logistic regression as described.

Table 3. Linear Regression on a Propensity-matched Sample Examining the Percentage Change in the Post-anesthesia Care Unit Length of Stay Associated with Neostigmine Versus Sugammadex Administration

\begin{tabular}{|c|c|c|c|c|}
\hline \multirow{2}{*}{$\begin{array}{l}\text { OLS regression on } \\
\text { the propensity-matched cohort } \\
\qquad(\mathrm{n}=1,444)\end{array}$} & \multicolumn{3}{|c|}{ Percentage change in PACU LOS and 95\% CI } & \multirow{2}{*}{$\begin{array}{c}\text { Univariable } \\
\text { P value } \\
\text { (Overall LR per ind. var. } \\
\text { /vs. reference category) }\end{array}$} \\
\hline & $\%$ Change & 95\% CI (low) & 95\% CI (high) & \\
\hline Reversal agent & & & & LR $P=0.701$ \\
\hline Neostigmine & Referent & Referent & Referent & Referent \\
\hline Sugammadex & $1.2 \%$ & $-5.0 \%$ & $7.9 \%$ & 0.702 \\
\hline Age (yr) & & & & LR $P=0.023$ \\
\hline Age at visit & $0.25 \%$ & $0.04 \%$ & $0.47 \%$ & 0.023 \\
\hline Sex & & & & LR $P=0.017$ \\
\hline Female & Referent & Referent & Referent & Referent \\
\hline Male & $-8.0 \%$ & $-14.1 \%$ & $-1.5 \%$ & 0.017 \\
\hline Weight & & & & LR $P=0.014$ \\
\hline log Weight (kg) & $17.37 \%$ & $3.26 \%$ & $33.40 \%$ & 0.014 \\
\hline Renal function category $\left(\mathrm{ml} / \mathrm{min} / \mathrm{m}^{2}\right)$ & & & & LR $P=0.036$ \\
\hline GFR $<30$ & Referent & Referent & Referent & Referent \\
\hline GFR 30-60 & $1.5 \%$ & $-10.3 \%$ & $14.9 \%$ & 0.813 \\
\hline GFR $>60$ & $-9.1 \%$ & $-18.0 \%$ & $0.81 \%$ & 0.071 \\
\hline ASA-PS & & & & LR $\mathrm{P}<0.001$ \\
\hline ASA-PS $1 / 2$ & Referent & Referent & Referent & Referent \\
\hline ASA-PS 3 & $5.1 \%$ & $-2.5 \%$ & $13.3 \%$ & 0.198 \\
\hline ASA-PS $4 / 5$ & $5.8 \%$ & $-7.0 \%$ & $20.3 \%$ & 0.396 \\
\hline ASA-PS Emergency (any) & $-24.6 \%$ & $-36.5 \%$ & $-10.5 \%$ & 0.001 \\
\hline Case length & & & & LR $\mathrm{P}<0.001$ \\
\hline log Case length (min) & $24.2 \%$ & $18.8 \%$ & $29.9 \%$ & $<0.001$ \\
\hline Procedure type & & & & LR $\mathrm{P}<0.001$ \\
\hline Cardio/thorac/vasc & Referent & Referent & Referent & Referent \\
\hline General/onc & $-4.6 \%$ & $-13.4 \%$ & $5.3 \%$ & 0.350 \\
\hline Neurosurgery & $15.1 \%$ & $3.1 \%$ & $28.5 \%$ & 0.013 \\
\hline Urology & $1.3 \%$ & $-9.6 \%$ & $13.4 \%$ & 0.827 \\
\hline Ob/gyn & $-4.3 \%$ & $-16.9 \%$ & $10.4 \%$ & 0.548 \\
\hline Gastroenterology & $-28.8 \%$ & $-40.0 \%$ & $-15.6 \%$ & $<0.001$ \\
\hline Other & $-19.2 \%$ & $-29.4 \%$ & $-7.6 \%$ & 0.002 \\
\hline
\end{tabular}

OLS: ordinary least-squares, PACU: post-anesthesia care unit, LR: likelihood ratio, ind var: independent variable, GFR: glomerular filtration rate, ASA-PS: American Society of Anesthesiologists physical status, LOS: length of stay, Cardio/thorac/vasc: cardiac, thoracic and vascular surgery, General/one: general and oncologic surgery, and oncologic surgery, Ob/gyn: obstetric and gynecologic surgery. 
rate of PACU MV due to rNMB decreased significantly, from 24 cases in P1 (0.63\%) to 7 in P2 (0.20\%; P = 0.005). MV because of other causes was not statistically significant. Sensitivity analysis performed with standard logistic regression yielded virtually identical results, with a significant change in PACU MV rate due to $\mathrm{rNMB}(\mathrm{P}=0.008)$, and none of the other causes yielded a statistically significant change in PACU MV (not shown).

\section{PACU LOS and emergence times}

We developed a propensity-matched cohort of patients from P2 who were reversed with either neostigmine or sugammadex ( $\mathrm{n}=1,444$ patients; characteristics summarized in Supplemental Appendix, Table S2). We performed linear regression to test for an independent association of sugammadex with a difference in PACU LOS and emergence time. The results of these analyses are presented in Tables 3 and 4, respectively. Owing to residual imbalance, factors independently associated with longer PACU LOS included female sex, increasing age, increasing weight, better renal function, non-emergency surgery, longer case length, and certain procedural categories. Factors associated with longer emergence times included longer surgical duration and certain procedural categories. No difference was observed in either outcome between reversal with neostigmine or reversal with sugammadex, in either the propensity-matched cohort or in the unadjusted cohort (not shown). We chose a genetic propensity-matching algorithm as it resulted in the best balance of preserved sample size and matched profile as measured by distance

Table 4. Linear Regression on a Propensity-matched Sample Examining the Percentage Change in the Emergence Time Associated with Neostigmine versus Sugammadex Administration

\begin{tabular}{|c|c|c|c|c|}
\hline \multirow{2}{*}{$\begin{array}{l}\text { OLS regression on } \\
\text { the propensity-matched cohort } \\
(\mathrm{n}=1,444)\end{array}$} & \multicolumn{3}{|c|}{ Percentage change in emergence time and $95 \% \mathrm{CI}$} & \multirow{2}{*}{$\begin{array}{c}\text { Univariable } \\
\text { P value } \\
\text { (Overall LR per ind. var } \\
\text { /vs. reference category) }\end{array}$} \\
\hline & $\%$ Change & 95\% CI (low) & 95\% CI (high) & \\
\hline Reversal agent & & & & LR $\mathrm{P}=0.194$ \\
\hline Neostigmine & Referent & Referent & Referent & Referent \\
\hline Sugammadex & $3.2 \%$ & $-1.6 \%$ & $8.2 \%$ & 0.196 \\
\hline Age $(y r)$ & & & & LR $\mathrm{P}=0.221$ \\
\hline Age at visit & $-0.10 \%$ & $-0.26 \%$ & $0.06 \%$ & 0.225 \\
\hline Sex & & & & LR $P=0.829$ \\
\hline Female & Referent & Referent & Referent & Referent \\
\hline Male & $-0.56 \%$ & $-5.5 \%$ & $4.7 \%$ & 0.830 \\
\hline Weight & & & & LR $P=0.089$ \\
\hline $\log$ Weight $(\mathrm{kg})$ & $-7.9 \%$ & $-16.4 \%$ & $1.3 \%$ & 0.091 \\
\hline Renal function category $\left(\mathrm{ml} / \mathrm{min} / \mathrm{m}^{2}\right)$ & & & & LR $P=0.195$ \\
\hline GFR $<30$ & Referent & Referent & Referent & Referent \\
\hline GFR $30-60$ & $-6.4 \%$ & $-14.7 \%$ & $2.7 \%$ & 0.163 \\
\hline GFR $>60$ & $-6.7 \%$ & $-13.6 \%$ & $0.8 \%$ & 0.078 \\
\hline ASA-PS & & & & LR $P=0.296$ \\
\hline ASA-PS $1 / 2$ & Referent & Referent & Referent & Referent \\
\hline ASA-PS 3 & $2.5 \%$ & $-3.1 \%$ & $8.4 \%$ & 0.394 \\
\hline ASA-PS $4 / 5$ & $9.8 \%$ & $-0.3 \%$ & $21.0 \%$ & 0.058 \\
\hline ASA-PS Emergency (any) & $4.5 \%$ & $-8.1 \%$ & $18.8 \%$ & 0.505 \\
\hline Case length & & & & LR $\mathrm{P}<0.001$ \\
\hline log Case length (min) & $5.9 \%$ & $2.4 \%$ & $9.5 \%$ & 0.001 \\
\hline Procedure type & & & & LR $\mathrm{P}<0.001$ \\
\hline Cardio/thorac/vasc & Referent & Referent & Referent & Referent \\
\hline General/onc & $-1.9 \%$ & $-8.9 \%$ & $5.5 \%$ & 0.599 \\
\hline Neurosurgery & $22.6 \%$ & $12.9 \%$ & $33.2 \%$ & $<0.001$ \\
\hline Urology & $-2.1 \%$ & $-10.1 \%$ & $6.6 \%$ & 0.628 \\
\hline Ob/gyn & $-3.8 \%$ & $-13.5 \%$ & $7.1 \%$ & 0.481 \\
\hline Gastroenterology & $-6.2 \%$ & $-17.5 \%$ & $6.6 \%$ & 0.326 \\
\hline Other & $-9.1 \%$ & $-17.9 \%$ & $0.5 \%$ & 0.063 \\
\hline
\end{tabular}

OLS: ordinary least-squares, LR: likelihood ratio, ind var: independent variable, GFR: glomerular filtration rate, ASA-PS: American Society of Anesthesiologists Physical Status, Cardio/thorac/vasc: cardiac, thoracic and vascular surgery, General/onc: general surgery and oncologic, Ob/gyn: obstetric and gynecologic surgery. 
reduction and balance improvement. The detected associations were not substantively different if alternative propensity-matching algorithms were employed. These sensitivity analyses were performed to assess for improved balance over the genetic algorithm and to ensure that the specific matching algorithm used was not a contributing factor to our findings.

\section{Discussion}

The introduction of sugammadex into unrestricted clinical use was associated with a significant reduction in PACU MV due to $\mathrm{rNMB}$, but no change was observed in the studied efficiency outcomes or overall utilization of MV in the PACU. Rates of PACU MV decreased from $0.63 \%$ to $0.20 \%$ following introduction of sugammadex. Prior descriptions of causes for PACU MV have been scant. We found the causes of PACU MV to be diverse, and rNMB accounted for $27.9 \%$ of PACU MV in P1 versus $11.7 \%$ in $\mathrm{P} 2$. The use of sugammadex likely enabled the reversal and extubation of patients who previously would have otherwise required postoperative MV.

\section{Impact of sugammadex availability on PACU and efficiency measures}

Broadly speaking, any rNMB carries a risk of patient discomfort, morbidity, and PPCs [8]. These complications have been well described, and a review of these complications, and the debate surrounding them, are beyond the scope of this report. We studied one of the more extreme consequence of rNMB, namely the need for mechanical ventilation in the PACU. When relying on anticholinesterases for NMBA antagonism, rNMB necessitating PACU MV can occur when a procedure is much shorter than expected, when deep NMB is required until case end, and in patients with unexpectedly prolonged TOF suppression. Related factors may include inexperience or errors with NMBA dosing and monitoring. Previous studies showing that sugammadex has the potential to eliminate $\mathrm{NMB}$ had not reported on its effect on PACU MV [5,23]. Olesnicky et al. [24] recently published an observational study of unrestricted sugammadex use in Australia, reporting a decrease in respiratory diagnoses with sugammadex availability. While they did observe a decrease in PACU MV, from $0.88 \%$ to $0.18 \%$, this was not statistically significant, perhaps because of the sample size.

Avoiding PACU MV reduces the workload for PACU staff (nurses, respiratory therapists, and physicians), hastens postoperative recovery, improves PACU efficiency, and avoids excess costs. These implications align with other work identifying earlier discharge readiness in patients who received sugammadex versus anticholinesterases for NMBA antagonism [25]. Although we studied a severe manifestation of rNMB, sugam- madex may hasten postoperative recovery and avoid more subtle types of PPCs [10,26,27]. Although we failed to identify improvements in the studied efficiency metrics, these and similar benefits should factor into pharmacoeconomic decisions regarding sugammadex deployment and implementation. Direct costs may be partially or fully offset through indirect savings in certain practice settings. PACU LOS and emergence time are highly complex, multifactorial outcomes that might be better assessed with a prospective observational design to standardize their assessment and allow more granular comparisons in future investigations.

As could be expected, sugammadex was not a panacea for all causes of rNMB. We identified prolonged NMB after succinylcholine administration, renal failure, and failure to administer sugammadex when appropriate as additional factors resulting in the need for PACU MV due to rNMB. Sugammadex is currently not recommended in patients with severe renal impairment owing to concerns related to delayed drug clearance and lack of safety data in this population, and for this reason, providers may rely on anticholinesterases for NMBA antagonism in this patient population [28]. Several studies of sugammadex use in patients with renal failure have demonstrated efficacy without major adverse events, but further study is required to determine whether sugammadex can indeed be used safely in this patient population $[26,29,30]$. Missed opportunities to prevent PACU MV via sugammadex administration may require education regarding the apparent risk/benefit ratio of sugammadex usage and the system-level improvement in care and value that accompanies avoidance of PACU MV [11].

PACU MV is a rare outcome, and our results should therefore be interpreted with caution, as the study may have been underpowered with regard to the primary outcome and MV-by-cause secondary outcome. Therefore, the findings related to rNMB, while intuitive, should still be considered exploratory. Our study is also subject to limitations inherent to retrospective designs, including the potential for unmeasured confounding. Linear regression of a propensity-matched cohort was used to reduce the selection bias from known covariates of interest in the analyzed sample. We attempted to minimize information bias with respect to misclassification of the causes of PACU MV by ensuring concurrence between multiple investigators. Although these determinations were made by retrospective chart review, a clear trail of evidence from the documentation was present in all cases reviewed. A form of confounding by indication cannot be excluded as patients with greater or lesser likelihood to develop rNMB may have been differentially represented in P1 and P2. This was at least partially addressed through the examination of relevant available covariates, such as case length and renal function. We also cannot fully exclude certain types of cyclic or seasonal influences. For example, the introduction of new train- 
ees into the operating room environment in July 2016 may have influenced the rates of PACU MV in P2. However, this would be expected to contribute, if anything, to an upward bias in MV related to $\mathrm{rNMB}$ resulting from the actions of inexperienced care providers in the period from July through October 2016 (P2).

In conclusion, the introduction of sugammadex did not decrease the rate of PACU MV, nor did it decrease the amount of time between surgery end and emergence. However, the introduction of sugammadex did reduce PACU MV secondary to rNMB, while other sources of PACU MV were statistically unchanged. Further work is needed to validate these findings and to understand the overall direct and indirect cost implications of the use of sugammadex rather than traditional pharmacologic methods for the reversal of NMBA.

\section{Acknowledgments}

We would like to thank Lisa Cogdill, Emory University Library and Information Technology, Atlanta, GA, USA, for her work and expertise in obtaining data from the Emory Healthcare Clinical Data Warehouse. We would like to thank George
Easton, PhD, Associate Professor of Information Systems \& Operations Management, Goizueta Business School, Emory University, Atlanta, GA, USA, for input into the statistical approach and assistance with interpretation of the results. We would like to thank Jim M. Blum, Emory University Department of Anesthesiology, for advice regarding the approach to the analysis and writeup.

\section{Funding Statement}

This research received no specific grant from any funding agency in the public, commercial, or not-for-profit sectors. This work was supported by the Emory University Department of Anesthesiology.

\section{ORCID}

Vikas N. O’Reilly-Shah, https://orcid.org/0000-0003-0741-0291

Grant C. Lynde, https://orcid.org/0000-0002-2055-3484

Craig S. Jabaley, https://orcid.org/0000-0001-6687-3953

\section{References}

1. Naguib M. Sugammadex: another milestone in clinical neuromuscular pharmacology. Anesth Analg 2007; 104: 575-81.

2. Lee C, Jahr JS, Candiotti KA, Warriner B, Zornow MH, Naguib M. Reversal of profound neuromuscular block by sugammadex administered three minutes after rocuronium: a comparison with spontaneous recovery from succinylcholine. Anesthesiology 2009; 110: $1020-5$.

3. Jones RK, Caldwell JE, Brull SJ, Soto RG. Reversal of profound rocuronium-induced blockade with sugammadex: a randomized comparison with neostigmine. Anesthesiology 2008; 109: 816-24.

4. Blobner M, Eriksson LI, Scholz J, Motsch J, Della Rocca G, Prins ME. Reversal of rocuronium-induced neuromuscular blockade with sugammadex compared with neostigmine during sevoflurane anaesthesia: results of a randomised, controlled trial. Eur J Anaesthesiol 2010; 27: 874-81.

5. Brueckmann B, Sasaki N, Grobara P, Li MK, Woo T, de Bie J, et al. Effects of sugammadex on incidence of postoperative residual neuromuscular blockade: a randomized, controlled study. Br J Anaesth 2015; 115: 743-51.

6. Carron M, Zarantonello F, Tellaroli P, Ori C. Efficacy and safety of sugammadex compared to neostigmine for reversal of neuromuscular blockade: a meta-analysis of randomized controlled trials. J Clin Anesth 2016; 35: 1-12.

7. Carron M, Baratto F, Zarantonello F, Ori C. Sugammadex for reversal of neuromuscular blockade: a retrospective analysis of clinical outcomes and cost-effectiveness in a single center. Clinicoecon Outcomes Res 2016; 8: 43-52.

8. Murphy GS, Brull SJ. Residual neuromuscular block: lessons unlearned. Part I: definitions, incidence, and adverse physiologic effects of residual neuromuscular block. Anesth Analg 2010; 111: 120-8.

9. Maybauer DM, Geldner G, Blobner M, Pühringer F, Hofmockel R, Rex C, et al. Incidence and duration of residual paralysis at the end of surgery after multiple administrations of cisatracurium and rocuronium. Anaesthesia 2007; 62: 12-7.

10. Abrishami A, Ho J, Wong J, Yin L, Chung F. Sugammadex, a selective reversal medication for preventing postoperative residual neuromuscular blockade. Cochrane Database Syst Rev 2009; (4): CD007362.

11. O'Reilly-Shah VN, Wolf FA, Jabaley CS, Lynde GC. Using a worldwide in-app survey to explore sugammadex usage patterns: a prospective observational study. Br J Anaesth 2017; 119: 333-5.

12. Ledowski T, Falke L, Johnston F, Gillies E, Greenaway M, De Mel A, et al. Retrospective investigation of postoperative outcome after reversal of residual neuromuscular blockade: sugammadex, neostigmine or no reversal. Eur J Anaesthesiol 2014; 31: 423-9.

13. Samad K, Khan M, Hameedullah, Khan FA, Hamid M, Khan FH. Unplanned prolonged postanaesthesia care unit length of stay and factors affecting it. J Pak Med Assoc 2006; 56: 108-12.

14. Gijsenbergh F, Ramael S, Houwing N, van Iersel T. First human exposure of Org 25969, a novel agent to reverse the action of rocuronium 
bromide. Anesthesiology 2005; 103: 695-703.

15. R Core Team. R: A Language and Environment for Statistical Computing [Internet]. Vienna: The R Foundation for Statistical Computing; 2017 Mar 6 [cited 2018 Mar 7]. Available from https://www.R-project.org/.

16. Li X, Wong W, Lamoureux EL, Wong TY. Are linear regression techniques appropriate for analysis when the dependent (outcome) variable is not normally distributed? Invest Ophthalmol Vis Sci 2012; 53: 3082-3.

17. Heinze G, Ploner M. logistf: Firth’s bias reduced logistic regression [Internet]. R package version. 2013. [cited 2018 Mar 7]. Available from https://rdrr.io/cran/logistf/man/logistf.html.

18. Heinze G, Schemper M. A solution to the problem of separation in logistic regression. Stat Med 2002; 21: 2409-19.

19. Puhr R, Heinze G, Nold M, Lusa L, Geroldinger A. Firth's logistic regression with rare events: accurate effect estimates and predictions? Stat Med 2017; 36: 2302-17.

20. Randolph JJ, Falbe K. A step-by-step guide to propensity score matching in R. Pract Assess Res Eval 2014; 19. Available from http:// pareonline.net/getvn.asp? $\mathrm{v}=19 \& \mathrm{n}=18$.

21. Ho DE, Imai K, King G, Stuart EA. MatchIt: nonparametric preprocessing for parametric causal inference. J Stat Softw 2011; $42: 1$-28.

22. Benoit K. Linear regression models with logarithmic transformations. Lond Sch Econ Lond 2011; 22: 23-36. Available from https://pdfs. semanticscholar.org/169c/c9bbbd77cb7cec23481f6ecb2ce071e4e94e.pdf.

23. Hristovska AM, Duch P, Allingstrup M, Afshari A. Efficacy and safety of sugammadex versus neostigmine in reversing neuromuscular blockade in adults. Cochrane Database Syst Rev 2017; 8: CD012763.

24. Olesnicky BL, Traill C, Marroquin-Harris FB. The effect of routine availability of sugammadex on postoperative respiratory complications: a historical cohort study. Minerva Anestesiol 2017; 83: 248-54.

25. Carron M, Zarantonello F, Lazzarotto N, Tellaroli P, Ori C. Role of sugammadex in accelerating postoperative discharge: A meta-analysis. J Clin Anesth 2017; 39: 38-44.

26. Staals LM, Snoeck MM, Driessen JJ, Flockton EA, Heeringa M, Hunter JM. Multicentre, parallel-group, comparative trial evaluating the efficacy and safety of sugammadex in patients with end-stage renal failure or normal renal function. Br J Anaesth 2008; 101: 492-7.

27. Groudine SB, Soto R, Lien C, Drover D, Roberts K. A randomized, dose-finding, phase II study of the selective relaxant binding drug, Sugammadex, capable of safely reversing profound rocuronium-induced neuromuscular block. Anesth Analg 2007; 104: 555-62.

28. Staals LM, Snoeck MM, Driessen JJ, van Hamersvelt HW, Flockton EA, van den Heuvel MW, et al. Reduced clearance of rocuronium and sugammadex in patients with severe to end-stage renal failure: a pharmacokinetic study. Br J Anaesth 2010; 104: 31-9.

29. de Souza CM, Tardelli MA, Tedesco H, Garcia NN, Caparros MP, Alvarez-Gomez JA, et al. Efficacy and safety of sugammadex in the reversal of deep neuromuscular blockade induced by rocuronium in patients with end-stage renal disease: a comparative prospective clinical trial. Eur J Anaesthesiol 2015; 32: 681-6.

30. Panhuizen IF, Gold SJ, Buerkle C, Snoeck MM, Harper NJ, Kaspers MJ, et al. Efficacy, safety and pharmacokinetics of sugammadex 4 mg kg-1 for reversal of deep neuromuscular blockade in patients with severe renal impairment. Br J Anaesth 2015; 114: 777-84. 


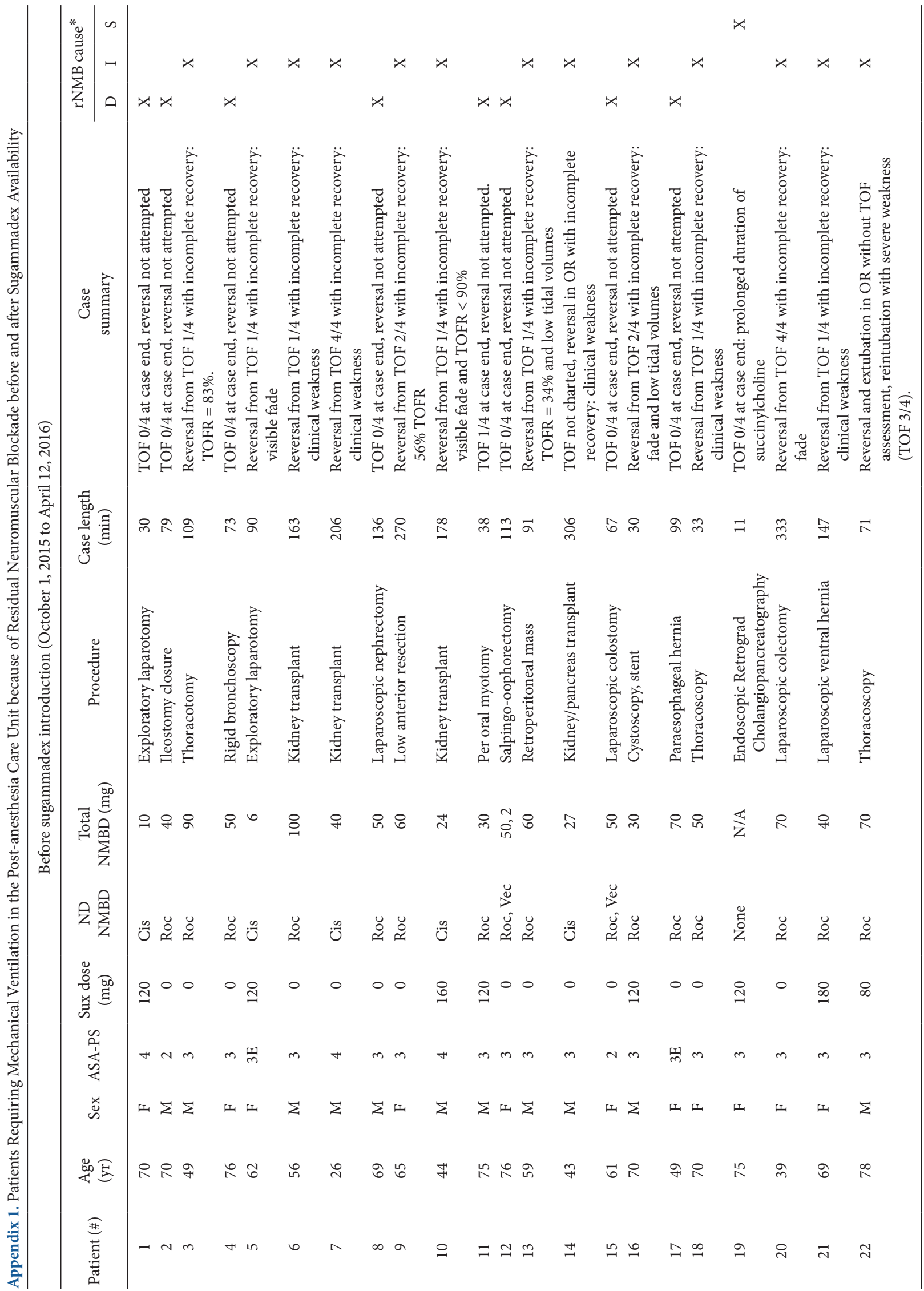




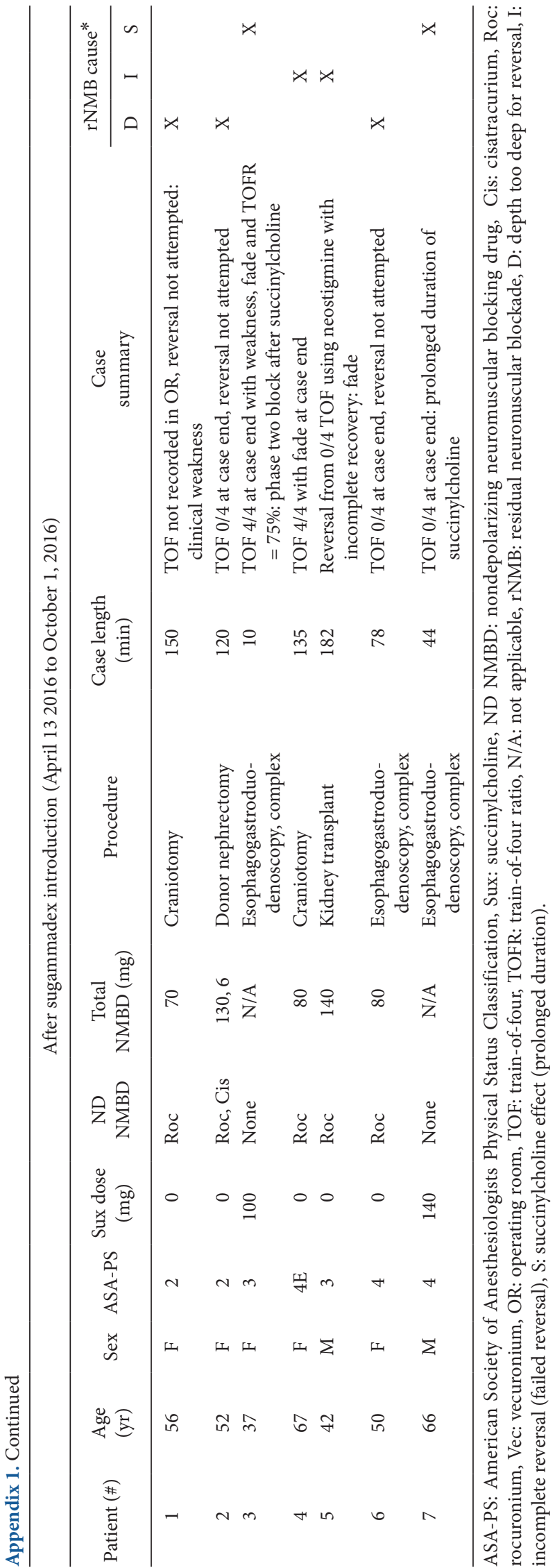

Appendix 2. Characteristics of Control Variables in the Propensity-matched Population to Compare the Emergence Time and Post-anesthesia Care Unit Length of Stay Between Patients Who Were Reversed Using Sugammadex versus Patients Who Were Reversed Using Neostigmine

\begin{tabular}{|c|c|c|}
\hline & Neostigmin & gammadex \\
\hline & & \\
\hline $\mathrm{N}$ & 876 & 1,773 \\
\hline distance & 0.42 & 0.29 \\
\hline AGE_AT_VISIT & 52.92 & 55.14 \\
\hline GENDER_DESCFemale & $58.7 \%$ & $55.8 \%$ \\
\hline GENDER_DESCMale & $41.3 \%$ & $44.2 \%$ \\
\hline WEIGHT_KG_Log & 4.37 & 4.39 \\
\hline ASA_Class3 & $55.6 \%$ & $55.2 \%$ \\
\hline ASA_Class $4 / 5$ & $10.5 \%$ & $6.5 \%$ \\
\hline ASA_ClassAny E & $4.8 \%$ & $3.3 \%$ \\
\hline GlomFiltRate_CatGFR $>60$ & $65.9 \%$ & $86.4 \%$ \\
\hline GlomFiltRate_CatGFR 30-60 & $13.0 \%$ & $12.1 \%$ \\
\hline SurgicalSpecialtyGeneral/Onc & $36.9 \%$ & $34.2 \%$ \\
\hline SurgicalSpecialtyNeurosurgery & $15.0 \%$ & $16.9 \%$ \\
\hline SurgicalSpecialtyUrology & $13.2 \%$ & $16.2 \%$ \\
\hline SurgicalSpecialtyOb/Gyn & $6.8 \%$ & $7.2 \%$ \\
\hline SurgicalSpecialtyGastroenterology & $3.8 \%$ & $6.7 \%$ \\
\hline SurgicalSpecialtyOther & $7.8 \%$ & $6.9 \%$ \\
\hline Log_SurgicalCaseLength & 4.71 & 4.63 \\
\hline & Propensity $\mathrm{N}$ & hed Cohort \\
\hline$\overline{\mathrm{N}}$ & 876 & 568 \\
\hline distance & 0.42 & 0.42 \\
\hline AGE_AT_VISIT & 52.92 & 53.30 \\
\hline GENDER_DESCFemale & $58.7 \%$ & $57.3 \%$ \\
\hline GENDER_DESCMale & $41.3 \%$ & $42.7 \%$ \\
\hline WEIGHT_KG_Log & 4.37 & 4.38 \\
\hline ASA_Class3 & $55.6 \%$ & $57.1 \%$ \\
\hline ASA_Class $4 / 5$ & $10.5 \%$ & $10.4 \%$ \\
\hline ASA_ClassAny E & $4.8 \%$ & $3.9 \%$ \\
\hline GlomFiltRate_CatGFR $>60$ & $65.9 \%$ & $66.2 \%$ \\
\hline GlomFiltRate_CatGFR 30-60 & $13.0 \%$ & $13.0 \%$ \\
\hline SurgicalSpecialtyGeneral/Onc & $36.9 \%$ & $37.6 \%$ \\
\hline SurgicalSpecialtyNeurosurgery & $15.0 \%$ & $14.6 \%$ \\
\hline SurgicalSpecialtyUrology & $13.2 \%$ & $13.2 \%$ \\
\hline SurgicalSpecialtyOb/Gyn & $6.8 \%$ & $7.1 \%$ \\
\hline SurgicalSpecialtyGastroenterology & $3.8 \%$ & $3.5 \%$ \\
\hline SurgicalSpecialtyOther & $7.8 \%$ & $7.8 \%$ \\
\hline Log_SurgicalCaseLength & 4.71 & 4.74 \\
\hline
\end{tabular}

ASA: American Society of Anesthesiologists Physical Status Classification, GFR: glomerular filtration rate, General/onc: general surgery and oncologic surgery, Ob/gyn: obstetric and gynecologic cases. 